\title{
Targeted Deep Sequencing Reveals Unrecognized KIT Mutation Coexistent with NFI Deficiency in GISTs
}

\author{
Jinchun $\mathrm{Wu}$ (D) $^{\prime}$ \\ Haiyan Zhou ${ }^{2}$ \\ Xiaoping $\mathrm{Yi}^{3}$ \\ Qiongzhi $\mathrm{He}^{4}$ \\ Tianxiang Lei $\mathbb{D}^{5}$ \\ Fengbo $\operatorname{Tan}^{5}$ \\ Heli Liu $\mathbb{D D}^{5}$ \\ Bin Li (D)
}

'Department of Oncology, Xiangya Hospital, Central South University, Changsha 410008, People's Republic of China; ${ }^{2}$ Department of Pathology, Xiangya Hospital, Central South University, Changsha 410008, People's Republic of China; ${ }^{3}$ Department of Radiology, Xiangya Hospital, Central South University, Changsha 410008, People's Republic of China; ${ }^{4}$ GeneplusBeijing Institute, Beijing, People's Republic of China; ${ }^{5}$ Department of General Surgery, Xiangya Hospital, Central South University, Changsha 410008, People's Republic of China
This article was published in the following Dove Press journal: Cancer Management and Research

Purpose: NF1-deficient GISTs account for about 1\% of gastrointestinal stromal tumors (GISTs) and are usually considered as a subtype of KIT/PDGFRA wild-type GISTs that have no detectable KIT and PDGFRA mutations. Some KIT/PDGFRA wild-type GISTs actually have cryptic $K I T$ mutations ( $m K I T)$. So we investigate whether concurrent $m K I T$ existed in NF1-associated GISTs.

Patients and Methods: Three independent cohorts were retrospectively analyzed. KIT/ PDGFRA wild-type GISTs in Xiangya Hospital between May 2017 and Oct 2019 were investigated by next-generation sequencing (NGS) approach targeted 1021 cancer-related genes regions. GISTs cases in Gene+ dataset from May 2017 to May 2020 were collected from the platform of this company. The genotypes of GISTs in MSKCC cohort were downloaded from cBioPortal.

Results: A total of 290 cases including 23 KIT/PDGFRA wild-type GISTs in Xiangya Hospital, 136 GISTs in Gene+ database, and 131 GISTs in MSKCC were enrolled. Twenty-six cases have NF1 mutations ( $m N F 1)$, and $48 \%(12 / 26)$ of NF1-mutated GISTs have concurrent mKIT. Compared with MSKCC $(2 / 10,20 \%)$, a higher ratio of $m K I T$ in NF1-associated GISTs was detected in Xiangya Hospital $(3 / 5,60 \%)$ and Gene+ $(7 / 11,64 \%)(\mathrm{p}<0.05)$. No mutation hotspot existed in $m N F 1$. Most of $m K I T$ centered within exon $11(7 / 12,58 \%)$ and others including exon $17(3 / 12,25 \%)$, exon $9(1 / 12,8 \%)$, exon $13(1 / 12,8 \%)$ and exon $21(1 / 12,8 \%)$. No differences in age, gender, and location were detected between NF1-related GISTs with $m K I T$ and those without $m K I T$. Three GIST cases of type I neurofibromatosis, skin neurofibromas and microGISTs $(\leq 1 \mathrm{~cm})$ were devoid of $m K I T$, but all the mini-GISTs $(1 \sim 2 \mathrm{~cm})$ and clinic GIST lesions $(>2 \mathrm{~cm})$ in two cases harbored $m K I T$.

Conclusion: $m K I T$ was not unusual in NF1-associated GISTs, especially in Chinese populations. The gain-of-function $m K I T$ possibly facilitated the progression of $N F 1$-deficient lesions to clinic GISTs, however, the underlying mechanism warrants further studies.

Keywords: gastrointestinal stromal tumor, NF1, KIT, deep sequencing

\section{Introduction}

Gastrointestinal stromal tumors (GISTs) are mesenchymal tumors usually located in the gastrointestinal tract. Most GISTs are sporadic and have oncogenic KIT or PDGFRA mutations. About 10-15\% GISTs lack KIT/PDGFRA mutation and are classified as KIT/ PDGFRA wild-type GISTs. ${ }^{1}$ GISTs are a heterogeneous group that has various entities including $S D H$-defective, $B R A F$-mutated, $N F 1$-deficient GISTs and other GISTs with rare gene abnormalities. ${ }^{1}$
Correspondence: Heli Liu; Bin Li Email heliliu@csu.edu.cn; bincsuxy@csu.edu.cn
Cancer Management and Research 2021:13 297-306 
Neurofibromin (NF1) gene encodes a protein named neurofibromin. It negatively regulates Ras family GTPases to maintain the inactive form ( $R A S-G D P)$ of $R A S$ oncoprotein. NF1 gene mutation causes defective neurofibromin protein that leads to loss of its inhibitory role on the activation of $R A S$ oncoprotein. ${ }^{2}$ Individuals with $N F 1$ deficiency carry a 34-fold risk than the average population for developing GIST, and GISTs occur in about $5-25 \%$ of cases diagnosed as type I neurofibromatosis. ${ }^{3,4}$

With the advent of the technology of new generation sequencing (NGS), occult $m N F 1$ was uncovered in about $60 \%$ quadruple-negative GIST, which suggested unrecognized NF1 deletion in clinical GISTs cases. ${ }^{5}$ Recently, NF1 somatic mutation was identified in a GIST patient without germline NF1 mutation, and somatic NF1 mutation in duodenal-jejunal flexure GIST frequently coexists with PDGFRA or $m K I T .^{6-8}$ About $22 \%$ (5/23) of cryptic $m K I T$ exists in KIT/PDGFRA/SDH/RAS-P wild-type GISTs, mostly within exon 11 and exon 9 of KIT, akin to the genotype of KIT in sporadic GISTs. ${ }^{1,9}$ Therefore, in this study, we retested our 23 archived Formalin-Fixed and Paraffin-Embedded (FFPE) samples of KIT/PDGFRA wildtype GISTs (negative of exons 9,11,13,14 and17 in KIT and exons 12, 14 and 18 in PDGFRA detected by routine Sanger sequencing) between May 2017 and Oct 2019 using targeted deep sequencing of 1021 cancer-related genes region. Meanwhile, we retrospectively analyze the profiles for the molecular status of genes aberration of GISTs by targeted deep sequencing with 73 genes panel or 1021 genes panel in Gene+ database and that of GISTs in MSKCC database using hybridization-capture-based sequencing with 410 genes that are druggable by approved therapies or are targets of experimental therapies. And then we make comparisons of the differences of gene landscapes between neurofibroma lesions in the skin and multiple lesions including micro- or mini-GISTs and GISTs more than $2 \mathrm{~cm}$ in intestines in three GIST cases with type I neurofibromatosis, aiming to investigate if $m K I T$ existed in $N F 1$-associated GISTs and possibly the pathogenesis of NF1-deficient GISTs.

\section{Patients and Methods}

\section{Patients and Samples}

The flowchart below illustrates the study design and patient screening process of this study (Figure 1). From May 2017 to Oct 2019, a total of 576 GISTs cases in our center (Xiangya Hospital) were screened. One hundred and eighty-five patients with no available genetic data of routine Sanger sequencing and 11 patients lost to follow-up were all excluded. 380 GISTs were included and 23 KIT/PDGFRA wild-type GISTs were identified and enrolled in this study. All clinical and pathological information was retrospectively collected, and samples were tested using next-generation sequencing (NGS) approach targeted 1021 cancer-related genes regions. The mutational data of 136 GISTs and 131 GISTs in the Gene+ dataset and MSKCC of the American population were accordingly extracted from the data platform of China National Genebank (www.cngb.org) or downloaded from cBioPortal (http://cbioportal.org/msk-impact). The clinical information including gender, age, location and survival data were also collected. Overall survival (OS) was determined from the date of diagnosis to the date of death or loss to follow-up. Time to progression (TTP) was defined from the date of surgery or stable disease to the date of relapse or disease progression. All cases diagnosed with GISTs in Xiangya Hospital were confirmed by an experienced pathologist who is a member of our multiple disciplinary tumor boards for GISTs. The study was approved by the Institutional Review Board (IRB) of Xiangya Hospital of Central South University, and the written informed consent of patients from Xiangya Hospital was waived. The patients included in the Gene+ dataset have been fully informed and signed the informed consent to perform sequencing and mutational analysis of their genetic data while their privacies are protected, according to the protocol approved by the Institutional Review Board of Geneplus-Beijing. The signed informed consent was not required for the patients of MSKCC dataset which belong to the cBioPortal database since it was an open-access database.

\section{Targeted Deep Sequencing and Data Collection}

The FFPE tissues of 23 KIT/PDGFRA wild-type GISTs were analyzed using targeted deep sequencing of 1021 cancerrelated genes region. Corresponding peripheral blood samples of these patients were collected as germline controls. The Next-Generation Sequencing was executed in accordance with the manufacturer's instructions (Illumina, San Diego, CA, USA). The somatic mutations of KIT/PDGFRA wild-type GISTs were investigated for the genomics landscape description with the ComplexHeatmap R package. The molecular status of gene aberration of 136 GISTs patients in the Gene+ database was detected by targeted deep sequencing with 73 genes panel or 1021 genes panel. The 131 cases of GISTs in the MSKCC database were detected for gene abnormalities using hybridization-capture-based sequencing 


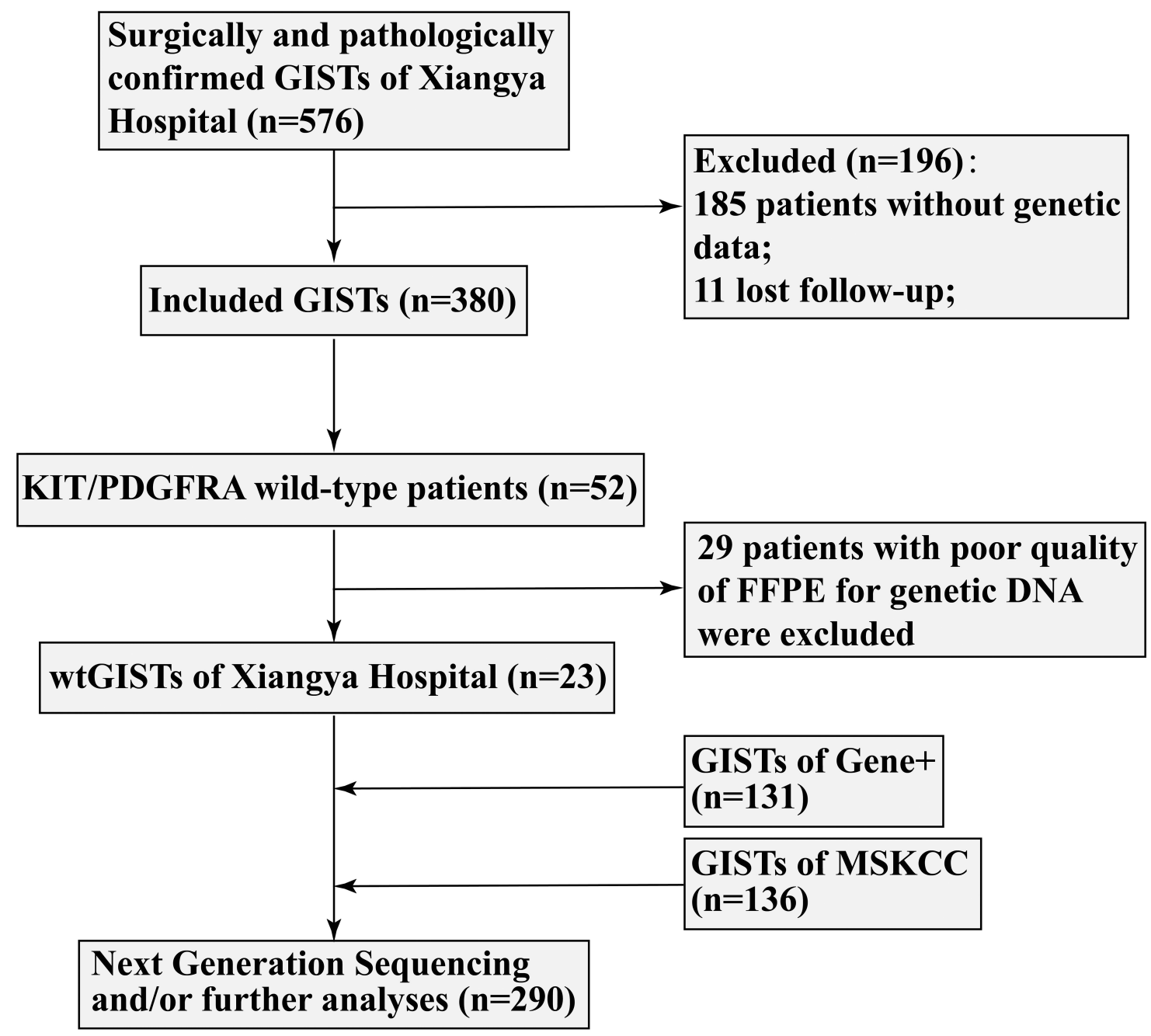

Figure I Case screening flow chart.

Notes: A total of 576 GISTs cases in our center (Xiangya Hospital) from May 2017 to Oct 2019 were screened. One hundred and eighty-five patients with no available genetic data of routine Sanger sequencing and II patients lost to follow-up were all excluded. Twenty-nine patients with poor quality FFPE were also excluded. Finally, 23 cases of 52 wild-types KIT/PDGFRA GISTs were included (negative for exons 9, II, I3, 14, and I7 in KIT and exon I2, I4, and I8 in PDGFRA using Sanger sequencing). A total of 136 GISTs cases between May 2017 to May 2020 in the Gene+ database and the 131 cases of GISTs from the MSKCC database were also included in our study.

with 410 genes that are druggable by approved therapies or are targets of experimental therapies.

\section{Statistical Analysis}

Numerical variables were summarized as the mean (standard deviation) and median (interquartile range). Categorical variables were reported as counts (percentage). The comparison between the categorical variables was analyzed by a chi-square test. The comparison between continuous variables was analyzed by a MannWhitney test. Survival analysis was assessed by the Kaplan-Meier method, and the differences were analyzed by the long-rank test. All tests of hypotheses were twotailed and conducted at a significance level of 0.05 .

\section{Results}

The Mutation Type of NFI in GISTs Totally 290 GISTs patients including 23 KIT/PDGFRA wild-type cases in Xiangya Hospital, 136 cases in Gene+ database and 131 cases in MSKCC database were enrolled in this study. The incidence of $m N F 1$ in KIT/PDGFRA wild-type GISTs of Xiangya Hospital was 23\% (5/23). The prevalence of $m N F 1$ in GISTs was about $8 \%$ both in the Gene+ database $(11 / 136)$ and in the MSKCC database (10/131). In the NF1-associated GISTs cases, 46\% (12/26) had $m K I T$. No difference in age, gender, and location was detected between the NF1-correlated GISTs with or without $m K I T$ (Table 1). The mutation status of NF1 is displayed in Figure 2. The most frequent mutant area was 
Table I Clinical Characteristics of GIST with NFI Mutation

\begin{tabular}{|c|c|c|c|}
\hline Clinical Features & $m N F I$ and $m K I T$ & $m N F I$ and $w t K I T$ & P-value (Methods) \\
\hline Number of patients $(\mathrm{N})$ & 12 & 14 & \\
\hline Age at diagnosis (years) & & & 0.6665 (Mann-Whitney) \\
\hline Mean \pm SD & $65.50 \pm 2.12$ & $44.00 \pm 22.63$ & \\
\hline Median & $65.5(36-68)$ & $44.00(28-94)$ & \\
\hline $\mathrm{NA}(\mathrm{N})$ & 2 & 8 & \\
\hline Gender $(\mathrm{N})$ & & & 0.2466 (Chi-square) \\
\hline Female & 5 & 9 & \\
\hline Male & 7 & 5 & \\
\hline NA & 0 & 0 & \\
\hline Location $(\mathrm{N})$ & & & 0.4389 (Chi-square) \\
\hline Duodenum & 1 & I & \\
\hline Small Bowel & 3 & 9 & \\
\hline Stomach & 4 & 3 & \\
\hline Intraabdominal & 0 & I & \\
\hline NA & 4 & 0 & \\
\hline
\end{tabular}

Abbreviations: NA, not available; N, number.

exon 28 (5/26, 19.2\%), followed by exon $3(4 / 26,15.4 \%)$ and exon $9(3 / 26,11.5 \%)$. No differences existed in the type of NF1 mutation between GISTs with KIT mutation and without KIT mutation (Table 2). Detailed descriptions of coexisting NF1 and KIT gene mutations in each of the 12 GISTs cases are shown in Table 3.

\section{Concurrent Gene Abnormalities in NFI-Deficiency GISTs}

Growing evidence showed that extra molecule events are required for GISTs which developed from NF1-deficient lesion or neurofibroma, ${ }^{10}$ so we analyze the genomic landscapes of 26 GIST patients with $m N F 1$. The most common abnormal genes concurrent with $m N F 1$ were KIT oncogene with a frequency of $46 \%$ and TP53 gene with a frequency of $15 \%$, followed by the mutations of other genes, including $\mathrm{NOTCH} 2, \mathrm{RB} 1$, and $\mathrm{TSC} 2$ et al, as shown in Supplementary 1.

\section{KIT Mutation in NFI-Associated GISTs}

Compared with the proportion of $m K I T$ in the American population in the MSKCC database $(20 \%, 2 / 10)$, a higher

\section{NF1 Exons}

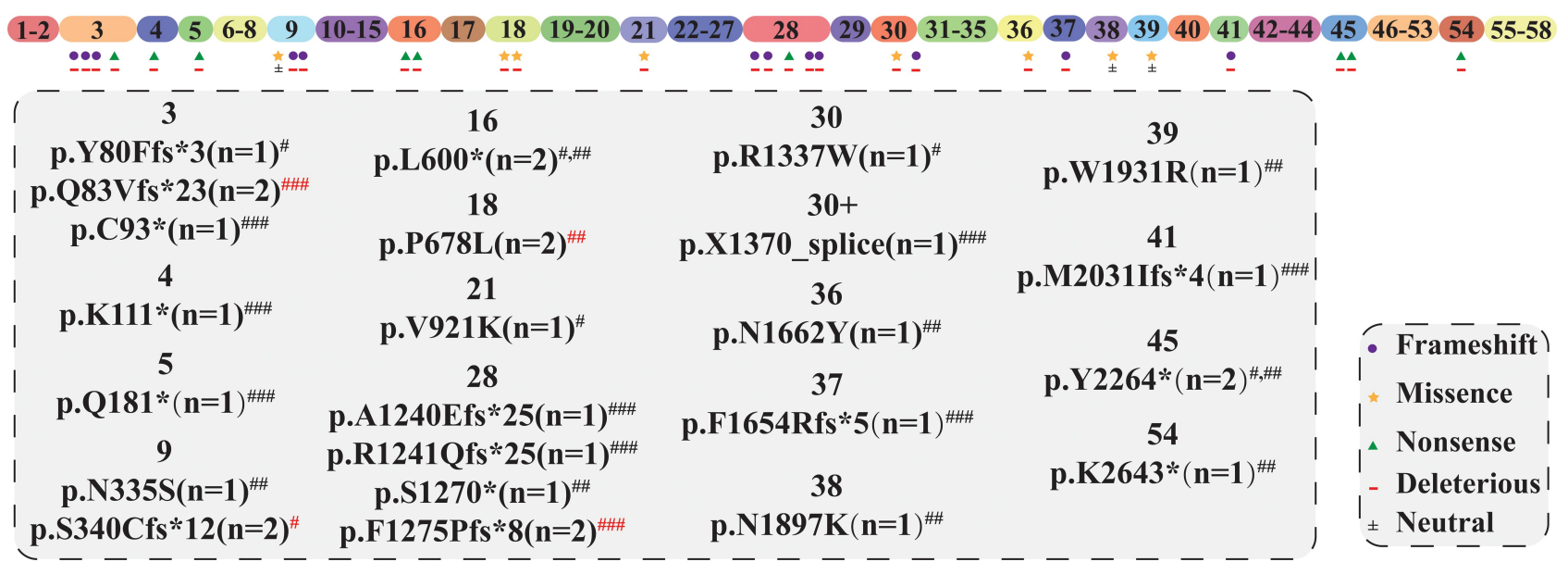

Figure 2 The mutation loci and subtypes within the NFI gene and their corresponding pathogenicity in GISTs.

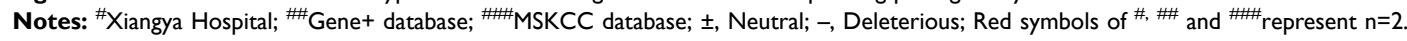


Table 2 The Mutation Types and Their Differences Between $m N F I$ with $m K I T$ and $m N F I$ with wtKIT

\begin{tabular}{|l|l|l|l|}
\hline mNFI Subtype & mKIT & wtKIT & P-value \\
\hline Frameshift & 6 & 9 & 0.4642 \\
Missense & 5 & 4 & 0.5229 \\
Nonsense & 3 & 3 & 0.8548 \\
\hline
\end{tabular}

Note: No differences of the type of NFI mutation between with KIT mutation and without KIT mutation.

ratio of $m K I T$ in $N F 1$-associated GISTs was detected in the Chinese cohort including that of our center $(60 \%, 3 / 5)$ and in the Gene+ database $(64 \%, 7 / 11)(\mathrm{p}<0.05)$ (Table 4). mKIT focus in the juxtamembrane domain (JM) or kinase domain showed hotspot mutation of KIT to be within exon 11 (7/12, $58 \%$ ), similar to the gene subtype of sporadic KIT-mutated GISTs. Other mutation locations of KIT were exon 17 (3/12, $25 \%)$, exon $9(1 / 12,8 \%)$, exon $13(1 / 12,8 \%)$, exon $21(1 / 12$, $8 \%)$ and exon $4(1 / 12,8 \%)$ respectively.

The mutation types of KIT contained in-frame deletions $(4 / 7,57 \%)$, deletion and insertion $(2 / 7,29 \%)$ and missense mutation $(1 / 7,14 \%)$ in exon $11, \mathrm{~N} 822 \mathrm{~K}$ in exon $17(3 / 3$,
$100 \%)$, A502_Y503dup in exon $9(1 / 1,100 \%)$ and I669_T670delinsMI in exon $13(1 / 1,100 \%)$. In the MSKCC dataset, both two cases of NF1-deficiency GISTs harbored double mutations, in which one of the shared mutations was still within the exon 11 of KIT (Figure 3).

\section{KIT Mutation in Various Lesions in Type I Neurofibromatosis}

With the purpose of exploring the role of KIT in the pathogenesis of $\mathrm{NF1-deficient} \mathrm{GISTs,} \mathrm{we} \mathrm{collected} \mathrm{various}$ samples range from skin neurofibromas, micro-GIST lesions $(\leq 1 \mathrm{~cm})$, mini-GIST lesions between $1 \mathrm{~cm}$ and 2 $\mathrm{cm}$, and GIST lesions $(>2 \mathrm{~cm})$ in three GISTs patients with type I neurofibroma. All these 3 cases had multiple café-au -lait spots and superficial neurofibromas which were considered as the diagnosis of type I Neurofibromatosis. Among the three cases, $m K I T$ existed in 2 cases with GISTs that were more than $2 \mathrm{~cm}$ but not in the skin neurofibromas subtype and micro-GISTs (Figure 4). Evidently, as shown in Figure 4, the number of gene

Table 3 Concurrent Mutation of NFI and KIT in GISTs Patients

\begin{tabular}{|c|c|c|c|c|c|c|}
\hline \multirow[t]{2}{*}{ Patient } & \multicolumn{3}{|l|}{ mNFI } & \multicolumn{3}{|l|}{$m K I T$} \\
\hline & cHGVS & Exon & Abundance & cHGVS & Exon & Abundance \\
\hline$I^{\#}$ & $\begin{array}{l}\text { c. } 239 \_245 \text { delATCTCTC } \\
\text { c. } 1017 \mathrm{CT}[2>1] \\
\text { c. } 6792 \mathrm{C}>\mathrm{A}\end{array}$ & $\begin{array}{l}3 \\
9 \\
45\end{array}$ & $\begin{array}{l}10 \% \\
3.60 \% \\
7.30 \%\end{array}$ & c. $2828 \mathrm{G}>\mathrm{T}$ & 21 & $4.30 \%$ \\
\hline $2^{\#}$ & c. $1799 \mathrm{~T}>\mathrm{A}$ & 16 & $38.40 \%$ & c. $2466 \mathrm{~T}>\mathrm{A}$ & 17 & $39.90 \%$ \\
\hline $3^{\#}$ & c. $4009 \mathrm{C}>\mathrm{T}$ & 30 & $3.50 \%$ & c. $2466 \mathrm{~T}>\mathrm{A}$ & 17 & $30.50 \%$ \\
\hline $4^{\# \#}$ & c.3805_3808dupGACT & 28 & $2.10 \%$ & c. $1676 \mathrm{~T}>\mathrm{G}$ & 11 & $39.90 \%$ \\
\hline $5^{\# \#}$ & c.7926dupT & 54 & $53.50 \%$ & c.1669_1674delTGGAAG & 11 & $33.10 \%$ \\
\hline $6^{\# \#}$ & c.569|T>G & 38 & $2.20 \%$ & c. $1669 \mathrm{~T}>\mathrm{A}$ & 11 & $40.80 \%$ \\
\hline $7^{\# \#}$ & c. $1004 \mathrm{~A}>\mathrm{G}$ & 9 & $40.70 \%$ & c.1504_I509dupGCCTAT & 9 & $49.90 \%$ \\
\hline $8^{\# \#}$ & c. $2033 \mathrm{C}>\mathrm{T}$ & 18 & $49.60 \%$ & c.1669_1674delTGGAAG & 11 & $11.50 \%$ \\
\hline $9^{\# \#}$ & c. $1799 \mathrm{~T}>\mathrm{A}$ & 16 & $38.80 \%$ & c. $2466 \mathrm{~T}>\mathrm{A}$ & 17 & $38.60 \%$ \\
\hline $10^{\# \#}$ & c. $2033 \mathrm{C}>\mathrm{T}$ & 18 & $50.20 \%$ & c.1676_1684delTTGTTGAGG & 11 & $34.30 \%$ \\
\hline $11^{\# \# \#}$ & $c .33 \mid \mathrm{A}>\mathrm{T}$ & 4 & NA & $\begin{array}{l}\text { c. } 698 \mathrm{G}>\mathrm{C} \\
\text { c. } 1657 \text { I662delTATGAA }\end{array}$ & $\begin{array}{l}4 \\
11\end{array}$ & $\begin{array}{l}\text { NA } \\
\text { NA }\end{array}$ \\
\hline $12^{\# \# \#}$ & c.4960_4967delTTTAAAAC & 37 & NA & $\begin{array}{l}\text { c.1668_1683delinsACAA } \\
\text { c.2007_2009delinsGAT }\end{array}$ & $\begin{array}{l}11 \\
13\end{array}$ & $\begin{array}{l}\text { NA } \\
\text { NA }\end{array}$ \\
\hline
\end{tabular}

Notes: The detailed information of mutated NFI gene and concurrent KIT mutation in each of the 12 GISTs cases; ${ }^{\#}$ Xiangya Hospital; ${ }^{\# /}$ Gene+ database; ${ }^{\#}$ MSKCC database. Abbreviations: NA, not available; cHGVS, ctDNA Human Genome Variation Society. 
Table 4 NFI Concurrent with KIT Mutation

\begin{tabular}{|l|l|l|l|l|}
\hline \multirow{2}{*}{ Genes } & \multicolumn{2}{|l|}{ Chinese (n,\%) } & $\begin{array}{l}\text { American } \\
\text { (n,\%) }\end{array}$ & $\begin{array}{l}\text { P-value } \\
\text { (Chi- } \\
\text { square) }\end{array}$ \\
\cline { 2 - 4 } & Xiangya & Gene+ & MSKCC & \\
\hline$m N F I$ and $m K I T$ & $3(I 3.0 \%)$ & $7(5.2 \%)$ & $2(1.5 \%)$ & 0.034442 \\
$m N F I$ and $w t K I T$ & $2(8.7 \%)$ & $4(2.9 \%)$ & $8(6.1 \%)$ & \\
\hline
\end{tabular}

mutations gradually increased with the clinical progression of neurofibromas to overt GISTS in all three cases.

\section{Survival of the Patients with mNFI and Those without mNFI}

At the time of drafting this paper, only one patient with wild-type $N F 1$ genotype had a recurrence, while all other patients recruited at our center still have stable disease. In the MSKCC dataset, the overall survival (OS) of the patients with wild-type $N F 1$ genotype showed shorter than that of the patients with NF1 mutation, but the difference in OS between these two groups had no statistical significance. (Supplementary 2).

\section{Discussion}

In this study, we investigated the frequency of $N F 1$ mutation in GISTs. The incidence of $m N F 1$ in KIT/ PDGFRA wild-type GISTs of Xiangya Hospital was $23 \%$. The prevalence of $m N F 1$ in GISTs in both Gene+ database and MSKCC database were $8 \%$, indicating no racial correlation. Among NF1-associated GISTs, $46 \%$ of cases had concurrent KIT oncogene mutation. In our center and Gene+ database, both of which had a Chinese population, $m K I T$ had a higher frequency of $60 \%(3 / 5)$ and $64 \%(7 / 11)$ in NF1-deficiency GISTs than that of the MSCKCC database with the American population $(20 \%, 2 / 10)(\mathrm{p}<0.05)$. No hotspot mutations were detected in $N F 1$, and there was no difference of mutation subtype in the $N F 1$ gene between those with or without mKIT. The mutation of KIT in NF1-associated GISTs centered on the JM or TKI domain, similar to that in the sporadic $m$ KIT GISTs. ${ }^{1}$

In the three GIST patients with type I neurofibromatosis, NGS was performed in a various range of neurofibromas lesions in the skin, micro-GISTs, or mini-GISTs and clinic GISTs more than $2 \mathrm{~cm}$ in the intestines. mKIT was not detected in neurofibromas of the skin and micro-GISTs, but it was identified in mini-GISTs and clinic GISTs bigger than $2 \mathrm{~cm}$ in two cases.

GISTs are mesenchymal tumors mostly located in the gastrointestinal tract. The majority of GISTs have KIT or PDGFRA mutations, and the rest about $10-15 \%$ harbor wild-type KIT/PDGFRA, hence considered as a heterogeneous entity mainly including $S D H$-defective and $R A S$-pathway abnormalities of $K / N / H-R A S, B R A F$, and $N F 1$ mutation. $N F 1$ is a tumor suppressor gene that negatively modulates $R A S$ signaling that accounts for less than $1 \%$ of total KIT/PDGFRA wild-type GISTs. ${ }^{1}$

Recently, using massively parallel sequencing, Gasparotto et al reported that about $60 \%$ (13/22) of quadruple-negative tumors harbored biallelic inactivation of $N F 1 .{ }^{5}$ In concordance with this, our center also disclosed about $22 \%$ of GIST patients have $m N F 1$ in KIT/PDGFRA wild-type GISTs.

\section{KIT Exons}

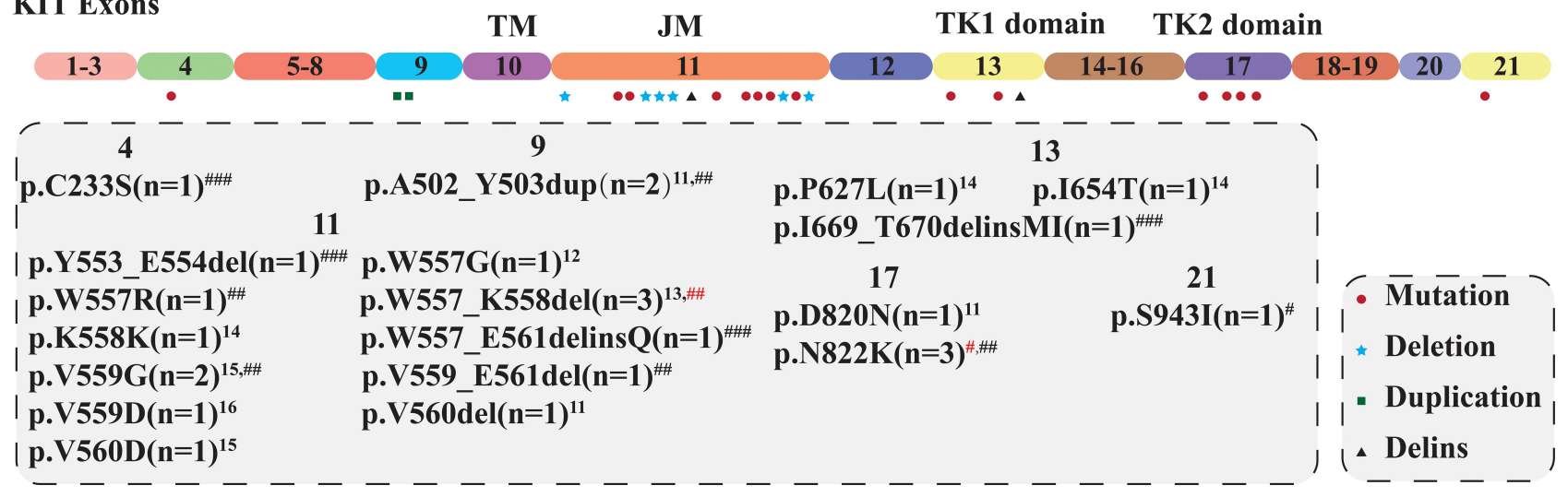

Figure 3 KIT mutations loci and subtype.

Notes: The distribution of various mutation subtypes identified by NGS within the exons of KIT gene; "Xiangya Hospital; ${ }^{\#}$ Gene+ database; ${ }^{\# \#}$ MSKCC database; \pm , Neutral; -, Deleterious; Red symbols of ${ }^{\# \#}$ and ${ }^{\# \#}$ represent $\mathrm{n}=2$.

Abbreviations: TM, transmembrane domain; JM, juxtamembrane domain; $\mathrm{N}$, number. 
A

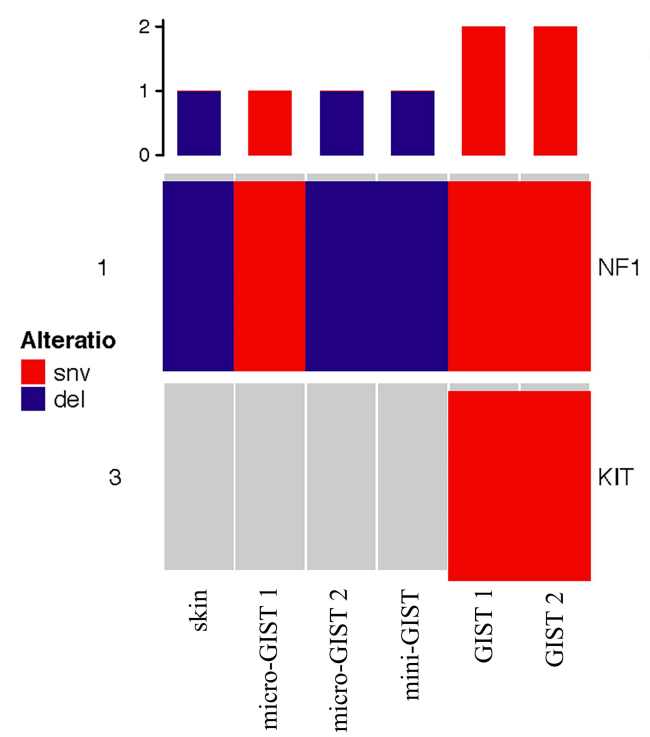

B

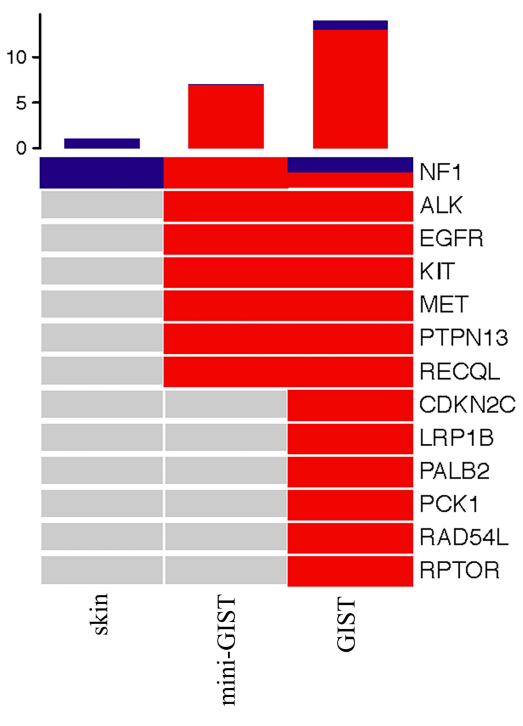

C

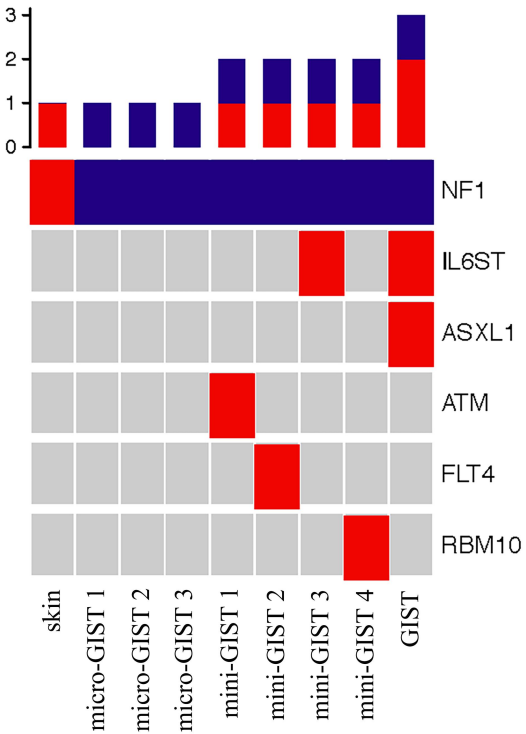

Figure 4 The genomic landscapes of skin neurofibroma and GISTs in 3 cases of type I neurofibromatosis.

Notes: (A-C) represent individual cases I-3, respectively. In panel A (case I) and panel B (case 2), compared with the lesions of skin neurofibromas and micro-GISTs ( $\leq$ I $\mathrm{cm}$ ) that merely harbor mNFI, GISTs lesions $(>2 \mathrm{~cm})$ have NFI aberration with a concurrent mKIT. In all three cases, the number of gene mutations is gradually increasing with the neurofibromas lesions to micro-GISTs or/and mini-GISTs to clinic GISTs.

$m K I T$ was first identified in GIST patients in 1998, leading to the understanding of the underlying mechanism of KIT-driven GISTs and exploration of abnormal genes that are concurrent with $m K I T{ }^{17}$ The first report about the molecular profile of $N F 1$-associated GISTs was provided by Kinoshita and colleagues. There was no $m K I T$ in their case series of 29 GISTs lesions in seven NF-1 patients; neither was there any $N F 1$ aberration in sporadic $m K I T$ GISTs. ${ }^{18,19}$

The mutual exclusion of the mutations of KIT and NFI in GISTs was reported in accumulative clinic studies. ${ }^{19}$ Indeed, haplodeficiency of neurofibromin was enough to promote the growth of a specific subtype of ICC expressing both KIT and S-100 protein in the small intestine, inferring that GIST progression in patients with type I Neurofibromatosis do not need gain-of-function mutation of KIT. $^{14}$

However, recent studies via targeted deep sequencing uncovered about $20 \%(5 / 26)$ have occult KIT abnormalities in a series of KIT/PDGFRA wild-type GISTs cases originally classified by Sanger sequencing, and 4 out of 5 cases were in the exon 11 of KIT. ${ }^{9}$ Similarly, Gao and colleagues reported that 19 out of 146 KIT/PDGFRA wildtype GISTs had the mutation of KIT according to NGS results, ${ }^{20}$ which was also mostly within the exon 11 of KIT $(14 / 19,74 \%)$. These results indicated that the incidence of $m K I T$ was underreported in the KIT/PDGFRA wild-type GISTs patients in the clinic when using conventional Sanger sequencing.

In nine cases of type I neurofibromatosis, Takazawa et al reported about $8 \%$ of $m K I T$ in 36 GIST lesions and all GISTs were of low-risk grade. ${ }^{10,14}$ Yantiss et al reported that three patients with $N F 1$ and multiple small intestinal stromal tumors were investigated and three tumors in different locations from one patient had identical point mutations in KIT exon 11, which has been previously reported in several studies of germline KIT mutation in JM or kinase domain developing GISTs. ${ }^{16}$ One NF1 case with all three GIST lesions had an identical mutation in KIT exon 11 with in-frame deletions. ${ }^{13}$ Mussi and colleagues detected 2 cases of 28 NF1-deficiency GISTs who had $m K I T$ with one of the cases of deletion in exon 11 and another one case of duplication in exon 9 in primary lesion. Intriguingly, one case in this report had exon 17 mutation of KIT in the metastatic lesion but not in the primary tumor. ${ }^{11}$

In our study, we found that $46 \%$ cases of $N F 1$-deficient GISTs harbored concurrent $m K I T$ in total. Two of the three patients in our center had the frequency of more than $30 \%$ of Exon 17 (p.N822K) as identified in NGS, which were negative for KIT mutation in the conventional Sanger method (detection of exons 9,11,13,14 and 17 in KIT and 
exon 12, 14 and 18 in PDGFRA). The possible explanation was tumor heterogeneity, as reported in the cases presented by Namgung and Bajor. ${ }^{12,25}$ Similar to our center, all the GISTs patients in the Gene+ database were of the Chinese population and had a higher frequency of $63 \%(10 / 16)$ of KIT mutation in NF1-deficiency GISTs than that of the American cohort in the MSCKCC database $(20 \%, 2 / 10)(p<0.05)$. The exact reason for this discrepancy is not clear. We speculate that racial and ethnic differences among the cohorts may play a role.

Apart from one of mKIT was in the extracellular domain of exon 4 in KIT, the others were within the region of JM or kinase domain. In line with the previous studies, ${ }^{21}$ the mutation in exon 11 was the most common subtype of $m K I T$, followed by exon 17, exon 9, exon 13, and exon 21. The mutation types of KIT contained inframe deletions, deletion and insertion, and missense mutation in exon 11, as well as point mutations in exon 17 and duplication in exon 9. In the MSKCC dataset, both two cases of NF1-deficiency GISTs harbored double mutations within KIT, one of the shared mutations was still within the exon 11 of KIT (Figure 3). These results uncovered that the mutation scenery of KIT in $N F 1$-associated GISTs was similar to that of sporadic $m K I T$ GISTs, hinting at the role of KIT in the development of NF1-aberrant GISTs, but it was still largely unknown.

KIT protein is a transmembrane type III receptor tyrosine kinase, consisting of an extracellular region and a cytoplasmic region which constitute a juxtamembrane domain and two kinase domains. Type I neurofibromatosis presents alterations in the $N F 1$ gene located in the long arm of chromosome 17 (17q11.2) and contains 50 exons, which code for the neurofibromin protein. GISTs linked to NF-1 frequently prove loss of heterozygosity at $14 \mathrm{q}$ and 22q similar to sporadic GISTs mutated by KIT and PDGFRA. ${ }^{22}$ Further study showed that the KIT ligand signal can stimulate the proliferation of neurofibrosarcoma cells. ${ }^{23}$ Heterozygous inactivation of the NF1 gene enhances $c$-kit-induced Ras output by reducing neurofibromin levels and RAS-GAP activity in cell lineages, which interprets the coordination mechanism underlying the $m K I T$ with deficient $N F 1$ for GIST progression. ${ }^{13,24}$ Namgung presented two GIST lesions of one type I neurofibromatosis patient with E11 mutation in KIT in the extramural portion of the largest tumor, but wtKIT in the intramural portion of the largest tumor and the other tumor. ${ }^{12}$ Secondary point mutation of D820N in exon 17 was shown in the metastatic lesion of one NF1 patient, but the primary tumor was wild type. ${ }^{11}$ Both of them suggested that $m K I T$ probably developed at a later step of NF1-deficient tumor genesis. ${ }^{25}$ This hypothesis was also observed in our study showing that $m K I T$ was in the miniGISTs more than $1 \mathrm{~cm}$, but not in micro-GISTs and skin neurofibromas. Further studies were required to depict the role and the mechanism of $m K I T$ in the pathogenesis of GISTs in some $N F 1$ patients. ${ }^{16,26}$

The treatment with imatinib in the GISTs associated with NF1 inactivation is ineffective and not recommended. ${ }^{1}$ Three NF1 cases with mKIT showed a poor reaction to imatinib treatment. ${ }^{11}$ Several cases showed that $N F 1$-associated GISTs have a good response to sunitinib or Regorafenib. ${ }^{27,28}$ So the optimal treatment in the subtype of concurrent mutations of NF1 and KIT remains to be elucidated. Besides, it is reported that the risk of recurrence and mortality is very similar between $N F 1$ and non-NF1 patients after surgical resection of GISTs. ${ }^{29}$ In accordance with this, most cases included in our study were post-surgery, and an adjuvant TKI regimen was given to these GIST patients with medium- to highrisk grade. The TTP and OS of these subgroups between NF1 deficiency and NF1-intact GISTs were similar. However, this conclusion remains equivocal due to the short follow-up period and the limited sample size.

This study has several limitations. Firstly, it was a retrospective study that is inherently limited by some confounding factors or bias, including clinical information. Secondly, NGS with different gene panels among these three cohorts from our center, Gene+, and MSKCC datasets affects the comparability of their differences. Thirdly, limited samples and a short follow-up time are inconclusive to show the effect of NF1 mutation on the survival of GIST cases.

\section{Conclusion}

Collectively, our study disclosed a high frequency of unrecognized $m K I T$ in $N F 1$-associated GISTs. Further underlying mechanisms of KIT oncogene cooperation with deficient NF1 in the pathogenesis of GISTs warrant further probing. NGS holds the potential for screening undiscovered $m K I T$ from a subset of GISTs originally regarded as KIT/PDGFRA wild-type tumors using regular Sanger sequencing. Due to its comparatively higher sensitivity and tumor heterogeneity, it provides a better understanding of mKIT in NF1-associated GISTs as well as identifying potentially eligible patients for optimal therapy. 


\section{Ethics Statement}

The retrospective study was approved by the Institutional Review Board (IRB) of Xiangya Hospital of Central South University, and informed consent of patients from Xiangya Hospital was waived (IRB No. 202,009,686). The signed informed consent was obtained from the patients of the Gene+ dataset, and not required for the patients of MSKCC dataset which belongs to the cBioPortal database since it was publicly available. The study was directed in accordance with the recommendations delineated in the World Medical Association Declaration of Helsinki. All personal data within this study were remained confidential and not for commercial use.

\section{Acknowledgment}

We want to give our thanks to Omar Abdihamid who check the words, grammar and English expression in the manuscript and attached legends.

\section{Funding}

This work was granted by the National Natural Science Foundation of China (81200366, 81572281, 81702278, 81974367 ) and Province Natural Science Foundation of Hunan (No. 14JJ6004) and the Key Subject Education Department of Hunan ([2012]594) and Scientific Research Project of Hunan Provincial Department of Education (No. 18K001).

\section{Disclosure}

Qiongzhi He is an employee of Geneplus-Beijing Institute. The authors declare that they have no other potential conflicts of interest for this work.

\section{References}

1. von Mehren M, Joensuu H. Gastrointestinal stromal tumors. J clin oncol. 2018;36(2):136-143. doi:10.1200/JCO.2017.74.9705

2. Philpott C, Tovell H, Frayling IM, Cooper DN, Upadhyaya M. The NF1 somatic mutational landscape in sporadic human cancers. Hum Genomics. 2017;11(1):13. doi:10.1186/s40246-017-0109-3

3. Giuly JA, Picand R, Giuly D, Monges B. Von Recklinghausen disease and gastrointestinal stromal tumors. Am J Surg. 2003;185(1):86-87. doi:10.1016/S0002-9610(02)01111-X

4. Uusitalo E, Rantanen M, Kallionpää RA, et al. Distinctive cancer associations in patients with neurofibromatosis type $1 . \mathrm{J}$ clin oncol. 2016;34(17):1978-1986. doi:10.1200/JCO.2015.65.3576

5. Gasparotto D, Rossi S, Polano M, et al. Quadruple-negative gist is a sentinel for unrecognized neurofibromatosis type 1 syndrome. Clin Cancer Res. 2017;23(1):273-282. doi:10.1158/1078-0432.CCR-16-0152

6. Belinsky MG, Rink L, Cai KQ, et al. Somatic loss of function mutations in neurofibromin 1 and $\mathrm{MYC}$ associated factor $\mathrm{X}$ genes identified by exome-wide sequencing in a wild-type GIST case. BMC Cancer. 2015;15:887. doi:10.1186/s12885-015-1872-y
7. Burgoyne AM, De Siena M, Alkhuziem M, et al. Duodenal-jejunal flexure gi stromal tumor frequently heralds somatic nf1 and notch pathway mutations. JCO Precision Oncol. 2017;2017:2017. doi:10.1200/PO.17.00014

8. Li K, Tjhoi W, Shou C, et al. Multiple gastrointestinal stromal tumors: analysis of clinicopathologic characteristics and prognosis of 20 patients. Cancer Manag Res. 2019;11:7031-7038. doi:10.2147/CMAR.S197560

9. Astolfi A, Indio V, Nannini $M$, et al. Targeted deep sequencing uncovers cryptic kit mutations in KIT/PDGFRA/SDH/RAS-P wild-type GIST. Front Oncol. 2020;10:504. doi:10.3389/ fonc. 2020.00504

10. Salvi PF, Lorenzon L, Caterino S, Antolino L, Antonelli MS, Balducci G. Gastrointestinal stromal tumors associated with neurofibromatosis 1: a single centre experience and systematic review of the literature including 252 cases. Int J Surg Oncol. 2013;2013:398570. doi: $10.1155 / 2013 / 398570$

11. Mussi C, Schildhaus HU, Gronchi A, Wardelmann E, Hohenberger P. Therapeutic consequences from molecular biology for gastrointestinal stromal tumor patients affected by neurofibromatosis type 1. Clin Cancer Res. 2008;14(14):4550-4555. doi:10.1158/1078-0432.CCR-080086

12. Namgung H. Gastrointestinal stromal tumor with KIT mutation in neurofibromatosis type 1. J Korean Surg Soc. 2011;81(4):276-280. doi:10.4174/jkss.2011.81.4.276

13. Cheng SP, Huang MJ, Yang TL, et al. Neurofibromatosis with gastrointestinal stromal tumors: insights into the association. Dig Dis Sci. 2004;49(7-8):1165-1169. doi:10.1023/B:DDAS.0000037806.14471.a2

14. Takazawa Y, Sakurai S, Sakuma Y, et al. Gastrointestinal stromal tumors of neurofibromatosis type I (von Recklinghausen's disease). Am J Surg Pathol. 2005;29(6):755-763. doi:10.1097/01. pas.0000163359.32734.f9

15. Tokunaga M, Nanjo S, Yoshita H, et al. Multiple synchronous sporadic gastrointestinal stromal tumors in the stomach and jejunum. Internal Medicine. 2018;57(12):1719-1723. doi:10.2169/internalmedicine.0229-17

16. Yantiss RK, Rosenberg AE, Sarran L, Besmer P, Antonescu CR. Multiple gastrointestinal stromal tumors in type I neurofibromatosis: a pathologic and molecular study. Modern Pathol. 2005;18 (4):475-484. doi:10.1038/modpathol.3800334

17. Mei L, Smith SC, Faber AC, et al. Gastrointestinal stromal tumors: the gist of precision medicine. Trends in Cancer. 2018;4(1):74-91. doi:10.1016/j.trecan.2017.11.006

18. Kinoshita K, Hirota S, Isozaki K, et al. Absence of c-kit gene mutations in gastrointestinal stromal tumours from neurofibromatosis type 1 patients. J Pathol. 2004;202(1):80-85. doi:10.1002/path.1487

19. Miettinen M, Fetsch JF, Sobin LH, Lasota J. Gastrointestinal stromal tumors in patients with neurofibromatosis 1: a clinicopathologic and molecular genetic study of 45 cases. Am J Surg Pathol. 2006;30 (1):90-96. doi:10.1097/01.pas.0000176433.81079.bd

20. Gao J, Li J, Li Y, et al. Intratumoral KIT mutational heterogeneity and recurrent KIT/PDGFRA mutations in KIT/PDGFRA wild-type gastrointestinal stromal tumors. Oncotarget. 2016;7(21):30241-30249. doi:10.18632/oncotarget.7148

21. Szucs Z, Thway K, Fisher C, et al. Molecular subtypes of gastrointestinal stromal tumors and their prognostic and therapeutic implications. Future Oncol. 2017;13(1):93-107. doi:10.2217/fon-2016-0192

22. Miettinen M, Lasota J, Sobin LH. Gastrointestinal stromal tumors of the stomach in children and young adults: a clinicopathologic, immunohistochemical, and molecular genetic study of 44 cases with long-term follow-up and review of the literature. Am J Surg Pathol. 2005;29(10):1373-1381. doi:10.1097/01.pas.0000172190.79552.8b

23. Badache A, Muja N, De Vries GHD. Expression of Kit in neurofibromin-deficient human Schwann cells: role in Schwann cell hyperplasia associated with type 1 neurofibromatosis. Oncogene. 1998;17(6):795-800. doi:10.1038/sj.onc.1201978 
24. Ryan JJ, Klein KA, Neuberger TJ, et al. Role for the stem cell factor/ KIT complex in Schwann cell neoplasia and mast cell proliferation associated with neurofibromatosis. $J$ Neurosci Res. 1994;37 (3):415-432. doi:10.1002/jnr.490370314

25. Bajor J. Gastrointestinal stromal tumors in neurofibromatosis type 1. Orv Hetil. 2009;150(4):149-153. doi:10.1556/oh.2009.28478

26. Shinomura Y, Kinoshita K, Tsutsui S, Hirota S. Pathophysiology, diagnosis, and treatment of gastrointestinal stromal tumors. $J$ Gastroenterol. 2005;40(8):775-780. doi:10.1007/s00535-005-1674-0

27. Kalender M, Sevinc A, Tutar E, Sirikci A, Camci C. Effect of sunitinib on metastatic gastrointestinal stromal tumor in patients with neurofibromatosis type 1: a case report. World $j$ Gastroenterol. 2007;13(18):2629-2632. doi:10.3748/wjg.v13.i18.2629
28. Fujimi A, Nagamachi Y, Yamauchi N, et al. Gastrointestinal stromal tumor in a patient with neurofibromatosis type 1 that was successfully treated with regorafenib. Internal Medicine. 2019;58(13):1865-1870. doi:10.2169/internalmedicine.2321-18

29. Nishida T, Tsujimoto M, Takahashi T, Hirota S, Blay JY, WatayaKaneda M. Gastrointestinal stromal tumors in Japanese patients with neurofibromatosis type I. J Gastroenterol. 2016;51(6):571-578. doi:10.1007/s00535-015-1132-6

\section{Publish your work in this journal}

Cancer Management and Research is an international, peer-reviewed open access journal focusing on cancer research and the optimal use of preventative and integrated treatment interventions to achieve improved outcomes, enhanced survival and quality of life for the cancer patient.
The manuscript management system is completely online and includes a very quick and fair peer-review system, which is all easy to use. Visit http://www.dovepress.com/testimonials.php to read real quotes from published authors. 